\title{
Plastocrono e número final de nós de cultivares de soja em função da época de semeadura
}

\author{
Plastochron and final node number of soybean cultivars as a function of sowing date
}

\author{
Juliano Dalcin Martins ${ }^{\mathrm{I}}$ Sidinei Zwick Radons ${ }^{\mathrm{II}}$ Nereu Augusto Streck ${ }^{\mathrm{II}}$ \\ Alberto Eduardo Knies ${ }^{\mathrm{I}}$ Reimar Carlesso $^{\mathrm{IV}}$
}

\section{RESUMO}

O plastocrono, intervalo de tempo entre o aparecimento de dois nós sucessivos na haste principal da planta, e o número final de nós (NFN) são importantes variáveis do desenvolvimento de plantas. $O$ objetivo deste trabalho foi determinar o plastocrono e o NFN de cultivares de soja (Glycine $\max (L$.$) Merrill) semeadas em diferentes épocas em um local$ de clima subtropical. Um experimento de campo foi realizado em Santa Maria, RS, no ano agrícola de 2005/2006, com três épocas de semeadura (09/11/2005, 09/12/2005 e 28/01/2006). Foram utilizadas 15 cultivares de soja recomendadas para as regiões Norte e Centro da Argentina, e Sul e Sudeste do Brasil. As variáveis medidas foram o número acumulado de nós (NN) e o NFN na haste principal. O plastocrono foi estimado pelo inverso do coeficiente angular da regressão linear entre o $N N$ e a soma térmica acumulada a partir de emergência (temperatura base $=10^{\circ} \mathrm{C}$ ). Semeaduras tardias resultaram em menor plastocrono e menor NFN, provavelmente devido ao menor fotoperíodo a que as plantas foram submetidas durante a fase de emissão de nós, uma resposta característica de plantas de dia curto.

Palavras-chave: Glycine max, plastocrono, número final de nós, soma térmica, fotoperíodo.

\section{ABSTRACT}

The plastochron, which is the time interval between the appearance of two successive nodes and the final node number (FNN) are important variables of the vegetative development in soybean. The objective of this study was to determine the plastochron and the FNN of soybean (Glycine max (L.) Merrill) cultivars sown in different dates under irrigated conditions in a subtropical location. A field experiment was carried out in Santa Maria, RS, Brazil during 2005/2006 growing season, with three sowing dates (09/11/2005, 09/12/ 2005 and 28/01/2006). It was used 15 soybean cultivars recommended for the Central and North Argentina, and South and Southeast Brazil. The variables measured were the number of nodes (NN) and the (FNN) on the main stem. The plastochron was estimated by the inverse of the slope of the linear regression between $N N$ and the thermal time (base temperature $=10^{\circ} \mathrm{C}$ ) accumulated since plant emergence. The late sowing resulted in lower plastochron and FNN, probably because of the lower photoperiod under which the plants were submitted in the node phase, a typical response of short day plants.

Key words: Glycine max, plastochron, final node number, thermal time, photoperiod.

\section{INTRODUÇÃO}

A soja (Glycine $\max$ (L.) Merrill) é a oleaginosa mais cultivada e o quarto grão mais produzido no mundo, após o milho, trigo e arroz. O Brasil é o segundo maior produtor mundial de soja, sendo cultivados no ano agrícola 2007/2008, aproximadamente, 21,3 milhões de hectares, resultando na produção de 60 milhões de toneladas (CONAB, 2008). Esses indicadores demonstram a importância desse cultivo tanto em escala mundial como no agronegócio brasileiro.

'Programa de Pós-graduação em Engenharia Agrícola, Centro de Ciências Rurais (CCR), Universidade Federal de Santa Maria (UFSM), Santa Maria, RS, Brasil.

IIUniversidade Federal da Fronteira Sul (UFFS), Campus Cerro Largo, RS, Brasil.

IIIDepartamento de Fitotecnia, CCR, UFSM, 97105-900, Santa Maria, RS, Brasil. E-mail: nstreck2@yahoo.com.br. Autor para correspondência.

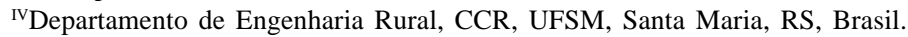


A unidade de tempo utilizada para caracterizar o desenvolvimento vegetal é a soma térmica ( ${ }^{\circ} \mathrm{C}$ dia) (STRECK et al., 2005). Essa unidade de tempo se baseia na premissa de que uma cultura necessita de uma determinada soma térmica acima de uma temperatura base para completar uma fase ou o seu ciclo (CAMARGO et al., 1987). A soma térmica é a medida de tempo biológico das plantas em substituição ao calendário civil, pois quantifica o efeito da temperatura sobre o desenvolvimento vegetal, o qual é um dos fatores ecológicos que governam o desenvolvimento das plantas, incluindo o aparecimento de nós e folhas (McMASTER \& SMIKA, 1988). Muitas cultivares de soja têm seu desenvolvimento influenciado, além da temperatura, pelo fotoperíodo, sendo classificadas como plantas de dia curto, ou seja, fotoperíodos decrescentes aceleram seu desenvolvimento (SINCLAIR et al., 2005; SETIYONO et al., 2007).

A velocidade de surgimento de folhas ou de nós na haste é um importante parâmetro do desenvolvimento vegetal. Ao integrar a velocidade de surgimento de folhas ou nós no tempo, tem-se o número de folhas ou de nós (NN) acumulados, respectivamente, os quais são uma excelente medida do desenvolvimento vegetal (XUE et al., 2004). Como cada folha é associada a um nó, o NN está diretamente relacionado com a evolução da área foliar, a qual é responsável pela interceptação da radiação solar usada na fotossíntese para produção de biomassa. Em soja, o NN é usado para caracterizar a fase vegetativa, definida como o período entre a emergência e o aparecimento do ultimo nó (SETIYONO et al., 2007). Durante seu ciclo, a planta passa por vários estágios, de V0 (nó cotiledonar) a Vn (último nó) e esta fase se sobrepõe parcialmente à fase reprodutiva, entre o aparecimento da primeira flor (R1) e a maturidade fisiológica (R8), ocorrendo aparecimento de nós durante parte da fase reprodutiva de R1 até o início da formação do grão (R5), momento em que é atingido o Vn (JOHNSON, 1997; SETIYONO et al., 2007).

O número final de nós (NFN) é outra importante variável de desenvolvimento, pois está diretamente relacionado com a duração do ciclo (SETIYONO et al., 2007). Quanto maior for o NFN, mais longa é a fase vegetativa e, consequentemente, mais prolongado é o ciclo de desenvolvimento da cultura (STRECK et al., 2003). Em soja, o NFN é atingido no estágio Vn e, como Vn coincide com R5, o valor do NFN é utilizado em alguns modelos de simulação do desenvolvimento (SETIYONO et al., 2007). Além disso, em cultivares de soja que respondem ao fotoperíodo, mudanças na duração do ciclo de desenvolvimento em resposta à variação do fotoperíodo acarretam mudanças no NFN, ou seja, o Vn depende do fotoperíodo (SETIYONO et al., 2007).
O NN em uma haste pode ser estimado a partir do conhecimento do tempo necessário para o aparecimento de dois nós sucessivos. Em dicotiledôneas, o intervalo entre o aparecimento de dois nós sucessivos na mesma haste é denominado plastocrono (SINCLAIR, 1984; STRECK et al., 2005). O conceito do plastocrono $\left({ }^{\circ} \mathrm{C}\right.$ dia) tem sido utilizado para estimar o $\mathrm{NN}$ em modelos de simulação do desenvolvimento de dicotiledôneas como a soja (SINCLAIR, 1986; STRECK et al., 2008).

$\mathrm{O}$ valor do plastocrono em soja pode variar com a cultivar (SINCLAIR et al., 2005; STRECK et al., 2008) e com o déficit hídrico no solo (STRECK et al., 2008). O NFN, por sua vez, pode variar entre cultivares, com a época de semeadura e com o fotoperíodo (SETIYONO et al., 2007). No Brasil, a soja é cultivada em regiões tropicais e subtropicais, existindo um grande número de cultivares e, anualmente, várias cultivares novas são disponibilizadas aos produtores. A caracterização de desenvolvimento dessas cultivares em diferentes regiões fisiográficas e épocas de semeadura é importante, contribuindo para melhoria da eficiência da cadeia produtiva da soja no Brasil (STRECK et al., 2008). O objetivo deste estudo foi determinar o plastocrono e o NFN em cultivares de soja semeadas em diferentes épocas em um local de clima subtropical.

\section{MATERIAL E MÉTODOS}

O experimento foi realizado a campo, na área experimental do Departamento de Engenharia Rural da Universidade Federal de Santa Maria, Santa Maria, RS, localizada na região fisiográfica da Depressão Central do Rio Grande do Sul (latitude 29 43 'S, longitude de $53^{\circ} 43^{\prime} \mathrm{W}$ e altitude de $\left.95 \mathrm{~m}\right)$. O clima local, segundo a classificação climática de Köppen, é Cfa, isto é, subtropical úmido, com verões quentes e sem estação seca definida (MORENO, 1961). O solo do local é um Argissolo Vermelho distrófico arênico, unidade de mapeamento São Pedro (EMBRAPA, 1999).

Foram realizadas semeaduras em três épocas durante o ano agrícola 2005/2006: 09/11/2005 (Época 1), 09/12/2005 (Época 2) e 28/01/2006 (Época 3), com emergência em 17/11/2005, 16/12/2005 e 04/02/2006, respectivamente. As duas primeiras épocas estão dentro do período de semeadura recomendado para a região (21/10 a 10/12) (REUNIÃO DE PESQUISA DE SOJA DA REGIÃO SUL, 2006), enquanto que a terceira época está fora do período recomendado. Foram usadas 15 cultivares de soja, utilizadas nas regiões Norte e Centro da Argentina, e Sul e Sudeste do Brasil (Tabela 1), com diferentes ciclos, origens e hábitos de crescimento. O delineamento experimental foi blocos ao acaso com três repetições. Cada parcela (unidade experimental) 
Tabela 1 - Ciclo de desenvolvimento, locais de recomendação, origem e hábito de crescimento das cultivares de soja utilizadas neste trabalho.

\begin{tabular}{|c|c|c|c|c|}
\hline Cultivar* & Ciclo & Recomendação & Origem & Háb. Cresc. \\
\hline 'MUNASQA' & Médio & Norte ARG & EEAOC-ARG & Determinado \\
\hline ‘N.K.48-00’ & Precoce & Centro ARG & Syngenta, ARG & Indeterminado \\
\hline 'N.K. 6.6’' & Tardio & Norte ARG & Syngenta, ARG & Determinado \\
\hline ‘N.K. 7.5’ & Médio & Norte ARG & Syngenta, ARG & Indeterminado \\
\hline ‘CD 219’ & Tardio & RS PR & COODETEC & Determinado \\
\hline 'CD 214' & Precoce & RS, SC, PR MS, SP & COODETEC & Determinado \\
\hline ‘CD 213’ & Precoce & RS, SC, PR MS, SP & COODETEC & Determinado \\
\hline ‘BRS Charrua’ & Tardio & RS & EMBRAPA & Determinado \\
\hline 'BRS 247’' & Médio & SC, PR, SP & EMBRAPA & Determinado \\
\hline 'BRS 245’ & Médio & SC, PR, SP & EMBRAPA & Determinado \\
\hline 'BRS 244’' & Médio & RS, SC, PR & EMBRAPA & Determinado \\
\hline ‘A 7053’' & Tardio & Norte ARG & Nidera, ARG & Determinado \\
\hline ‘A 8100’' & Tardio & Norte ARG & Nidera, ARG & Determinado \\
\hline ‘M-SOY 7979’ & Precoce & RS, SC, PR, SP, MS & Monsanto & Determinado \\
\hline ‘M-SOY 8080’ & $* *$ & $* *$ & Monsanto & Determinado \\
\hline
\end{tabular}

*Todas as cultivares utilizadas são resistentes ao herbicida glifosato (RR). **Dados não obtidos. EEAOC = Estación Experimental Agroindustrial Obispo Colonbres. COODETEC = Cooperativa Central de Pesquisa Agrícola. EMBRAPA = Empresa Brasileira de Pesquisa Agrícola. ARG = Argentina. Fonte: REUNIÃO DE PESQUISA DE SOJA DA REGIÃO SUL (2006), MONSANTO (2008), INTA (2008).

constituiu cinco fileiras de cultivo de $3 \mathrm{~m}$ de comprimento espaçadas em $0,45 m$ entre si.

A emergência foi considerada quando $50 \%$ das plântulas estavam visíveis acima do nível do solo. Em três plantas por parcela, foram contados, três vezes por semana, os nós na haste principal, considerando um nó visível quando os bordos do limbo do folíolo central do trifólio, associado a esse nó, não mais se tocaram (JOHNSON, 1997). No final do experimento, determinou-se o NFN na haste principal das plantas identificadas.

A irrigação foi determinada com base na estimativa da evapotranspiração de referência $\left(\mathrm{ET}_{0}\right)$ pelo método de Penman-Monteith, com os valores de coeficientes de cultura recomendados por ALLEN et al. (1998). Irrigações foram aplicadas quando a evapotranspiração máxima indicava valores acumulados de $32 \mathrm{~mm}$.

A temperatura mínima e máxima diária do ar durante o período experimental foi obtida na Estação Climatológica Principal, pertencente ao 8oㅗ Distrito de Meteorologia do Instituto Nacional de Meteorologia, localizada a aproximadamente $500 \mathrm{~m}$ da área experimental. A temperatura média diária do ar (Tmed) foi calculada pela média aritmética das temperaturas máxima e mínima do ar. A soma térmica diária (STd, ${ }^{\circ} \mathrm{C}$ dia) foi calculada assumindo o valor de temperatura base (Tb) de $10^{\circ} \mathrm{C}$ (WANG et al., 1987; STRECK et al., 2008) por: STd = (Tmed - Tb). 1 dia. A soma térmica acumulada (STa, ${ }^{\circ} \mathrm{C}$ dia) a partir da emergência foi calculada por meio do somatório dos valores de STd, isto é, STa $=\Sigma$ STd.

Foi realizada a análise de regressão linear simples, obtendo-se equação de regressão linear entre os valores de STa e o NN na haste principal da planta (média das plantas por parcela). Tomando-se o inverso do coeficiente angular da equação de regressão linear simples, foi calculado o plastocrono. Os valores de plastocrono e NFN foram submetidos à análise de variância (ANOVA) em nível de 5\% de probabilidade, segundo um esquema bifatorial (15 cultivares $x 3$ épocas de semeadura) e as médias foram testadas pelo teste de Tukey em nível de 5\% de probabilidade.

\section{RESULTADOS E DISCUSSÃO}

Durante o período de emissão de folhas, a média de temperatura média diária do ar foi $25,0{ }^{\circ} \mathrm{C}$, $25,2^{\circ} \mathrm{C} \mathrm{e} 23,5^{\circ} \mathrm{C}$, respectivamente, para as semeaduras de 09/11/2005, 09/12/2005 e 28/01/2006. Dois dias (29/3 e 30/3/2006) apresentaram temperaturas médias mais baixas $\left(17,5\right.$ e $\left.17,0^{\circ} \mathrm{C}\right)$, porém acima da Tb. Na maioria dos dias, a temperatura média do ar foi inferior à temperatura ótima da cultura $\left(30^{\circ} \mathrm{C}\right)$, com exceção de três dias (8/1, 15/01 e 16/01/2006) em que Tmed foi superior a $30^{\circ} \mathrm{C}$. O fotoperíodo variou de 15 horas no dia 21/12/2005 a 12 horas no último dia de emissão de nós.

A regressão linear entre o NN e o STa foi significativa em todas as épocas de semeadura e 
cultivares, com coeficiente de determinação $\left(\mathrm{r}^{2}\right)$ acima de 0,96 . Essa alta relação linear indica que a temperatura do ar é o principal elemento meteorológico que influencia a emissão de nós em soja (SINCLAIR, 2005; STRECK et al., 2008).

A ANOVA para a variável plastocrono indicou efeito significativo dos fatores principais e da interação deles. O quadrado médio foi maior para época de semeadura $(1054,4)$ do que para cultivar $(33,0)$, o que indica que o plastocrono foi mais afetado pelo ambiente do que pelo genótipo. A análise estatística foi desdobrada e está apresentada na tabela 2. Na época 1 , o maior plastocrono $\left(69,3^{\circ} \mathrm{C}\right.$ dia nó $\left.{ }^{-1}\right)$ foi da cultivar 'BRS 247', não diferindo das cultivares 'BRS 244', 'N.K. 6.6', 'N.K. 48-00', ‘A 7053' e ‘BRS 245' e o menor plastocrono $\left(55,5^{\circ} \mathrm{C}\right.$ dia nó $\left.{ }^{-1}\right)$ foi da cultivar MUNASQA, que não diferiu apenas da cultivar 'BRS 247'. Nas épocas 2 e 3, as cultivares não diferiram entre si, com média de 51,3 e $50,3^{\circ} \mathrm{C}$ dia nó ${ }^{-1}$, respectivamente. Nas épocas avaliadas, o plastocrono decresceu com o atraso da semeadura, sendo maior na época 1 para a maioria das cultivares, exceto para 'CD 214' e 'M-SOY 7979’, que não diferiram entre as épocas. O plastocrono foi menor na época 3 para a maioria das cultivares, exceto para ‘N.K. 6.6', 'N.K. 48-00', ‘A8100’ e ‘M-SOY 7979’, quando foi maior que na época 2 (Tabela 2).

A média geral do plastocrono obtida nesse estudo foi de $53,5^{\circ} \mathrm{C}$ dia nó-1 ${ }^{-}$, mesmo valor obtido em SINCLAIR et al. (2005) como média de oito cultivares.
Também com cultivares brasileiras, STRECK et al. (2008) obtiveram médias de $55,9^{\circ} \mathrm{C}$ dia nó ${ }^{-1}$ (seis cultivares) em um ano mais seco e $50,7^{\circ} \mathrm{C}$ dia nó ${ }^{-1}$ (cinco cultivares) em um ano mais úmido. STRECK et al. (2008), estudando o plastocrono das cultivares 'CD 213', 'CD 214' e 'CD 219', observaram valores de plastocrono, respectivamente, de $48,1,50,2$ e $50,8^{\circ} \mathrm{C}$ dia nó ${ }^{-1}$, enquanto que no presente estudo os valores de plastocrono destas cultivares foram na média de 52,6, 52,3 e 53,4 $4^{\circ} \mathrm{C}$ dia nó ${ }^{-1}$.

A variação de plastocrono observada entre algumas cultivares tem implicações práticas. Considerando as cultivares ‘BRS 247’ e MUNASQA, que apresentaram valores médios de plastocrono para as três épocas de 57,0 e $50,3^{\circ} \mathrm{C}$ dia nó ${ }^{-1}$, respectivamente (Tabela 2), tem-se uma diferença de $6,7^{\circ} \mathrm{C}$ dia nó ${ }^{-1}$. Supondo que essas cultivares emitam 20 nós, com uma STd de $15^{\circ} \mathrm{C}$ dia nó ${ }^{-1}$, tem-se aproximadamente 9 dias de diferença entre a data de emissão do vigésimo nó na haste principal. Essa diferença é ainda maior considerando-se o cultivo na época 1 , em que a diferença de plastocrono foi maior $\left(13,5^{\circ} \mathrm{C}\right.$ dia nó $\left.{ }^{-1}\right)$ a favor da cultivar 'BRS 247'. Para as épocas 2 e 3, as diferenças de plastocrono entre cultivares não foram estatisticamente significativas.

O NFN também apresentou interação significativa entre cultivares e épocas de semeadura (Tabela 3). O quadrado médio foi maior para épocas de semeadura $(609,6)$ do que para cultivares $(8,34)$, o que

Tabela 2 - Plastocrono ( ${ }^{\circ} \mathrm{C}$ dia nó ${ }^{-1}$ ) na haste principal de 15 cultivares em três épocas de semeadura. Santa Maria, RS, $2005-2006$.

\begin{tabular}{|c|c|c|c|c|c|}
\hline \multirow{2}{*}{ Cultivar } & \multicolumn{4}{|c|}{-------------------------------------Época de semeadura'------------------------------------ } & \multirow{2}{*}{ DP das medias } \\
\hline & Época 1 & Época 2 & Época 3 & Média & \\
\hline 'BRS 247’ & $69,3 \mathrm{Aa}$ & $54,5 \mathrm{Ab}$ & $47,5 \mathrm{Ac}$ & 57,1 & 11,1 \\
\hline 'BRS 244’ & $62,4 \mathrm{ABa}$ & $58,0 \mathrm{Aab}$ & $50,6 \mathrm{Ab}$ & 57,0 & 6,0 \\
\hline 'N.K. 6.6’' & $61,7 \mathrm{ABa}$ & $52,8 \mathrm{Ab}$ & $53,0 \mathrm{Ab}$ & 55,8 & 5,1 \\
\hline 'N.K. 48-00' & 59,1 ABa & 49,6 Ab & 54,1 Aab & 54,2 & 4,8 \\
\hline ‘A 7053’’ & 59,7 ABa & $51,4 \mathrm{Ab}$ & 49,7 Ab & 53,8 & 5,0 \\
\hline 'BRS 245’ & $60,4 \mathrm{ABa}$ & $51,1 \mathrm{Ab}$ & 49,7 Ab & 53,4 & 5,8 \\
\hline ‘CD 219’ & $58,5 \mathrm{Ba}$ & $50,9 \mathrm{Ab}$ & $50,7 \mathrm{Ab}$ & 53,4 & 4,5 \\
\hline ‘N.K 7.5’ & $57,1 \mathrm{Ba}$ & 51,8 Aab & $48,4 \mathrm{Ab}$ & 52,6 & 3,3 \\
\hline 'M-SOY 8080' & $58,1 \mathrm{Ba}$ & 51,5 Aab & $48,4 \mathrm{Ab}$ & 52,6 & 5,0 \\
\hline 'CD 213’' & $58,9 \mathrm{Ba}$ & $50,3 \mathrm{Ab}$ & $48,7 \mathrm{Ab}$ & 52,6 & 5,5 \\
\hline 'BRS charrua' & $57,2 \mathrm{Ba}$ & 50,6 Aab & 49,5 Ab & 52,4 & 4,2 \\
\hline ‘A 8100’ & $58,2 \mathrm{Ba}$ & $48,5 \mathrm{Ab}$ & $50,4 \mathrm{Ab}$ & 52,4 & 5,1 \\
\hline ‘CD 214’ & $54,7 \mathrm{Ba}$ & $52,7 \mathrm{Aa}$ & 49,6 Аа & 52,3 & 2,6 \\
\hline 'M-SOY 7979' & $55,0 \mathrm{Ba}$ & 48,4 Аa & $51,0 \mathrm{Aa}$ & 51,5 & 3,3 \\
\hline 'MUNASQA' & $55,5 \mathrm{Ba}$ & $47,9 \mathrm{Ab}$ & $47,4 \mathrm{Ab}$ & 50,3 & 4,5 \\
\hline Média & 59,1 & 51,3 & 50,3 & 53,5 & 4,5 \\
\hline DP das medias & 3,6 & 2,6 & 1,8 & & \\
\hline CV \% & \multicolumn{5}{|c|}{6,48} \\
\hline
\end{tabular}

Médias seguidas pela mesma letra maiúscula na vertical e minúscula na horizontal não diferem entre si pelo teste de Tukey a $5 \%$ de probabilidade de erro. ${ }^{1}$ Época 1 = semeadura em 09/11/2005, Época 2 = semeadura em 09/12/2005 e Época 3 = semeadura em 28/01/2006. 
Tabela 3 - Número final de nós (nós planta ${ }^{-1}$ ) na haste principal de 15 cultivares em três épocas de semeadura. Santa Maria, RS, $2005-2006$.

\begin{tabular}{|c|c|c|c|c|c|}
\hline \multirow{2}{*}{ Cultivar } & \multicolumn{4}{|c|}{ - } & \multirow{2}{*}{ DP das médias } \\
\hline & Época 1 & Éроса 2 & Éроса 3 & Média & \\
\hline 'BRS 247' & $19,0 \mathrm{Ba}$ & $17,3 \mathrm{Aa}$ & 14,7 ABCa & 17,0 & 2,2 \\
\hline 'BRS 244’ & $19,7 \mathrm{Ba}$ & 17,3 Аа & 14,3 АВСa & 17,1 & 2,7 \\
\hline ‘N.K. 6.6’ & 20,3 Ba & 18,0 Aab & $12,3 \mathrm{Cb}$ & 16,9 & 4,1 \\
\hline 'N.K. 48-00’ & $19,7 \mathrm{Ba}$ & 16,0 Аа & 14,3 АВСа & 16,7 & 2,7 \\
\hline ‘A 7053’ & 21,3 ABa & $18,0 \mathrm{Aa}$ & $12,0 \mathrm{Cb}$ & 17,1 & 4,7 \\
\hline ‘BRS 245’ & $20,7 \mathrm{ABa}$ & 17,0 Aab & 14,3 Abc & 17,3 & 3,2 \\
\hline ‘CD 219’ & $22,0 \mathrm{ABa}$ & 20,3 Аа & 17,3 Аа & 19,9 & 2,4 \\
\hline 'N.K 7.5’ & $24,7 \mathrm{Aa}$ & $17,7 \mathrm{Ab}$ & $13,3 \mathrm{ABCb}$ & 18,6 & 5,7 \\
\hline 'M-SOY 8080’ & 23,0 ABa & 18,7 Aab & $16,7 \mathrm{ABa}$ & 19,4 & 3,2 \\
\hline ‘CD 213’' & $21,3 \mathrm{ABa}$ & $18,0 \mathrm{Aab}$ & $14,0 \mathrm{ABCb}$ & 17,8 & 3,7 \\
\hline 'BRS charrua' & 22,0 ABa & 18,3 Aab & $15,3 \mathrm{ABCb}$ & 18,6 & 3,3 \\
\hline ‘A 8100’' & $22,3 \mathrm{ABa}$ & 18,0 Aab & 13,0 BCb & 17,8 & 4,7 \\
\hline 'CD 214' & $22,0 \mathrm{ABa}$ & 19,7 Aab & 13,7 ABCb & 18,4 & 4,3 \\
\hline ‘M-SOY 7979’ & $24,0 \mathrm{ABa}$ & 19,3 Aab & $12,0 \mathrm{Cc}$ & 18,4 & 6,0 \\
\hline 'MUNASQA' & 21,3 ABa & 18,3 Аа & $15,7 \mathrm{ABCa}$ & 18,4 & 2,8 \\
\hline Média & 21,6 & 18,1 & 14,2 & 18,0 & 3,4 \\
\hline DP das medias & 1,6 & 1,1 & 1,6 & & \\
\hline CV \% & & & 5,96 & & \\
\hline
\end{tabular}

Médias seguidas pela mesma letra maiúscula na vertical e minúscula na horizontal não diferem entre si pelo teste de Tukey a $5 \%$ de probabilidade de erro. ${ }^{1}$ Época 1 = semeadura em 09/11/2005, Época 2 = semeadura em 09/12/2005 e Época 3 = semeadura em 28/01/2006.

indica que o NFN foi mais afetado pelo ambiente do que pelo genótipo. Na época 1 , o maior NFN foi observado para a cultivar 'N.K. 7.5' (24,7 nós planta $\left.{ }^{-1}\right)$, diferindo das cultivares 'N.K. 48-00', 'N.K. 6.6', 'BRS 244' e 'BRS 247', e o menor NFN foi da cultivar 'BRS 247’ (19 nós planta ${ }^{-1}$ ), que diferiu apenas da cultivar 'N.K. 7.5'. Na época 2, não houve diferença de NFN entre cultivares, com uma média de 18,1 nós planta ${ }^{-1} \mathrm{e}$, na época 3, as cultivares se diferenciaram em três grupos, com maior NFN na cultivar 'CD 219' (17,3 nós planta $^{-1}$ ) e menor na cultivar 'A 7053' e 'M-SOY 7979' (12 nós planta-1 ${ }^{-1}$. Entre as épocas avaliadas, o NFN decresceu com o atraso da semeadura, sendo estatisticamente maior na época 1 para oito das 15 cultivares usadas. O NFN foi menor na época 3 para as cultivares ‘A7053’ e ‘BRS 245' e ‘M-SOY 7979’ e, para as demais, o NFN não diferiu para a época 2 ou 1 (Tabela 3). As cultivares apresentaram resposta distinta quanto à redução do NFN nas diferentes épocas de semeadura. A cultivar 'M-SOY 7979' apresentou uma redução de 12 nós planta ${ }^{-1}$, enquanto a 'BRS 247’ teve uma redução de 4,3 nós planta ${ }^{-1}$ da primeira para a última época de semeadura.

A diminuição no NFN também foi observada com o atraso da semeadura em 12 cultivares de soja por SETIYONO et al. (2007) e, segundo esses autores, a diminuição está associada com a resposta ao fotoperíodo, quando a soja é cultivada em regiões extratropicais. Nessas regiões, semeaduras realizadas na metade da primavera, como para a época 1, expõem a planta de soja a fotoperíodos crescentes logo após a emergência até o solstício de verão, quando o fotoperíodo é máximo (15 horas no local do presente estudo), diminuindo a taxa de desenvolvimento, prolongando a fase vegetativa e aumentando o NFN. De semeaduras realizadas no final da primavera e no verão destas regiões, como as épocas 2 e 3, resultam plantas de soja expostas a fotoperíodos decrescentes logo após a emergência, reduzindo o NFN (SETIYONO et al., 2007). Assim, os resultados na tabela 3 indicam que as 15 cultivares de soja usadas no presente estudo apresentam resposta fotoperiódica. Diferenças no NFN entre as épocas 1 e 2 variaram com a cultivar e estão associadas a diferenças de resposta fotoperiódica, ou seja, quanto maior a redução no NFN com o atraso da época de semeadura, maior a resposta fotoperiódica da cultivar (SETIYONO et al., 2007).

Neste trabalho, observou-se a redução do plastocrono com o atraso da semeadura (Tabela 2), isto é, a velocidade de aparecimento de nós aumentou em semeaduras tardias, embora a redução do plastocrono com o atraso da semeadura tenha sido menos pronunciada do que a redução no NFN nas épocas 2 e 3 (Tabela 3). Apesar de não ser considerado o efeito do fotoperíodo sobre a velocidade de emissão de nós em soja (SETIYONO et al., 2007), é possível que a redução do plastocrono com o atraso da semeadura seja uma resposta ao fotoperíodo, já que o aumento da taxa de 
desenvolvimento em fotoperíodos decrescentes é uma típica resposta de planta de dia curto. Assim, a duração do ciclo de desenvolvimento em semeaduras tardias pode ser resultado do efeito combinado da redução do NFN e da diminuição do plastocrono.

\section{CONCLUSÃO}

O plastocrono e o número final de nós na haste principal em soja variam com a cultivar e a resposta genotípica dessas duas variáveis do desenvolvimento depende da época de semeadura. Semeaduras mais tardias resultam em menor plastocrono e número final de nós, provavelmente em resposta ao fotoperíodo a que as plantas são expostas.

\section{REFERÊNCIAS}

ALLEN, R.G. et al. Crop evapotranspiration: guidlines for computing crop water requirements. Roma: FAO-56, 1998. 300p.

CAMARGO, M.B.P. et al. Temperatura base para cálculo dos graus-dia para cultivares de soja em São Paulo. Pesquisa Agropecuária Brasileira, v.22, n.2, p.115-121, 1987.

CONAB. SOJA - BRASIL. Série histórica de área plantada. Disponível em: <http://www.conab.gov.br/conabweb/download/ Safra/SojaSerieHist.xls>. Online. Acesso em: 09 dez. 2008.

EMBRAPA. Sistema brasileiro de classificação de solos Brasília: Embrapa Produções de informações, 1999. 412p.

INTA. Boletín Informativo n.1 Proyecto cereales y oleaginasas. Disponível em: <http://www.inta.gov.ar/lasbrenas/ info/documentos/pv/cereales_oleaginosas1.pdf $>$. Online. Acesso em: 08 abr. 2009

JOHNSON, S.R. How a soybean plant develops. Ames: Iowa Stale University of Science and Technology, and Cooperative Extension Service, 1997. 18p. (Special Report n.53).

McMASTER, G.S.; SMIKA, D.E. Estimation and evaluation of winter wheat phenology in the central Great Plains. Agricultural and Forest Meteorology, v.43, n.1, p.1-18, 1988.

MONSANTO. Sementes/Soja. Disponível em: <http:// www.monsanto.com.br/ sementes/monsoy/biotec_presente/ Biotec_presente.asp>. Online. Acesso em: 08 dez. 2008.

MORENO, J.A. Clima do Rio Grande do Sul. Porto Alegre: Secretaria da Agricultura, Diretoria de Terras e colonização, Secção de geografia, 1961. 46p.

REUNIÃO DE PESQUISA DE SOJA DA REGIÃO SUL. Indicações técnicas para a cultura da soja no Rio Grande do Sul e em Santa Catarina 2006/2007. Pelotas: Embrapa Clima Temperado, 2006. 237p.

SETIYONO, T.D. et al. Understanding and modeling the effect of temperature and daylength on soybean phenology under high-yield conditions. Field Crops Research, v.100, p.257271, 2007. Disponível em: <http://www.sciencedirect.com/ science?_ob=MImg\&_imagekey=B6T6M-4KV8TD7-2 - 21\&_cdi=5034\&_user=687358\&_pii $=$ S0378429006001560\&_o rigin $=$ browse $\&$ zone $=$ rslt_list_item $\&$ _coverDate $=02 / 01 /$ $2007 \&$ \& s k = $998999997 \&$ w c h p = d G L b V l z zSkWA\&md5=1d7a7e8f955bdbf7aad0adacedb91da2\&ie=/ sdarticle.pdf $>$. Acesso em: 11 mar. 2011. doi: 10.1016/ j.fcr.2006.07.01

SINCLAIR, T.R. Leaf area development in field grown soybean. Agronomy Journal, v.76, n.1, p.141-146, 1984. Disponível em: <https://www.soils.org/publications/aj/abstracts/76/1/141N2>. Acesso em: 11 mar. 2011. doi: 00021962007600010034x.

SINCLAIR, T.R. Water and nitrogen limitations in soybean grain productivity. I. Model development. Field Crops Research, v.15, n.2, p.125-141, 1986. Disponível em: <http:/ /www.sciencedirect.com/science/article/B6T6M-48YVSSD-BK/ 2/cb5161c0d2cf 5fc6ad4b7ff3a15117e6>. Acesso em: 11 mar. 2011. doi: 10.1016/0378-4290(86)90082-1.

SINCLAIR, T.R. et al. Comparison of vegetative development in soybean cultivars for low-latitude environment. Field Crops Research, v.92, n.1, p.5359, 2005. Disponível em: <http://www.sciencedirect.com/ science?_ob=MImg\&_imagekey=B6T6M-4DH2D65-1$1 \&$ \&di $=5034 \&$ \&user $=687358 \&$ \& or i g i n = g a te wa y \&_cover D a t e $=04 / 14 /$ $2005 \&$ \& $k=999079998 \&$ view $=c \& w c h p=d G L z V t z-$ zSkWb\&md5=957429df122f1ef5297f77a97bfe7910\&ie=/ sdarticle.pdf $>$. Acesso em: 11 mar. 2011. doi: 10.1016/ j.fcr.2004.08.008.

STRECK, N.A. et al. Improving predictions of developmental stages in winter wheat: a modified Wang and Engel model. Agricultural and Forest Meteorology, v.115, n.3-4, p.139150, 2003. Disponível em: <http://www.sciencedirect.com/ science?_ob=MImg\&_imagekey=B6V8W-47DT8TW-11P\&_cdi=5881\&_user=687358\&_pii=S0168192302002289\& origin $=$ g a te w a y \& c o v e r D a t e $=03 / 30$ $2003 \&$ \& $\mathrm{s}=998849996 \& \mathrm{view}=\mathrm{c} \& \mathrm{w} \mathrm{chp}=\mathrm{dGLbVlz}$ zSkzk\&md5=b11ba0cc0f5ebf0b2331b06960322823\&ie=/ sdarticle.pdf $>$. Acesso em: 11 mar. 2011. doi: 10.1016/S01681923(02)00228-9.

STRECK, N.A. et al. Estimativa do plastocrono em meloeiro (Cucumis melo L.) cultivado em estufa plástica em diferentes épocas do ano. Ciência Rural, v.35, n.6, p.1275-1280, 2005. Disponível em: <http://www.scielo.br/pdf/cr/v35n6/ a08v35n6.pdf $>$. Acesso em: 11 mar. 2011. doi: 10.1590/ S0103-84782005000600008.

STRECK, N.A. et al. Estimativa do plastocrono em cultivares de soja. Bragantia, v.67, n.1, p.67-73, 2008. Disponível em <http://www.scielo.br/pdf/brag/v67n1/a08v67n1.pdf. Acesso em: 11 mar. 2011. doi: 10.1590/S0006-87052008000100008.

WANG, J. et al. A data base for predicting soybean phenology. Biotronics, v.16, p.25-38, 1987. Disponível em: <https:// qir.kyushu-u.ac.jp/dspace/bitstream/2324/8131/1/ KJ00004506730.pdf>. Acesso em: 10 mar. 2011.

XUE, Q. et al. Predicting leaf appearance in field grown winter wheat: evaluating linear and non-linear models. Ecological Modelling, v.175, p.261-270, 2004. Disponível em: <http:/ www.sciencedirect.comscience?_ob=MImg\&_imagekey=B6VBS4BJX1HYJR\&_cdi $=5934 \&$ _user $=687358 \&$ \&ii $=$ S0304380003004800\&_origin= gateway\&_coverDate=07152004\&_sk=998249996\&view=c\&wchp=dGLzVzzzSkWb\&md5=8de99c1e523d2b623fd5f29dd053c8f9\&ie =/ sdarticle.pdf $>$. Acesso em: 11 mar. 2011. doi: 10.1016/ j.ecolmodel.2003.10.018. 